\title{
Synthesis of Isoxazolopyridines and Spirooxindoles under Ultrasonic Irradiation and Evaluation of Their Antioxidant Activity
}

\author{
Emel Pelit \\ Faculty of Art and Sciences, Department of Chemistry, Kirklareli University, Kayali Campus, 39100 Kirklareli, Turkey \\ Correspondence should be addressed to Emel Pelit; emelpelit@yahoo.com
}

Received 20 June 2017; Accepted 8 August 2017; Published 12 October 2017

Academic Editor: Mohamed Afzal Pasha

Copyright (C) 2017 Emel Pelit. This is an open access article distributed under the Creative Commons Attribution License, which permits unrestricted use, distribution, and reproduction in any medium, provided the original work is properly cited.

New polycyclic-fused isoxazolo[4,5-e]pyridines and spirooxindoles were obtained via multicomponent reaction of 5-amino-3methylisoxazole, indan-1,3-dione, and aromatic aldehydes and reaction of 5-amino-3-methylisoxazole, isatin, and $\beta$-diketones in the presence of $( \pm$ )-camphor-10-sulfonic acid as an effective and nontoxic organocatalyst under ultrasound-promoted conditions. The antioxidant activity of the novel synthesized compounds was studied.

\section{Introduction}

Multicomponent reactions allow the building of several new bonds in a single step and therefore these one-pot reactions are one of the most attractive topics in synthetic chemistry [1]. These reactions have enormous effectiveness, particularly for the construction of heterocyclic compounds which exhibit a wide range of biological activities [2-5]. Multicomponent reactions proceed in accordance with green chemistry principles in terms of a high degree of atom economy, easier progress of reactions, decreased reaction times, lack of waste products, and low power consumption $[6,7]$.

Isoxazole and spirooxindole compounds have exhibited many biological properties such as antibacterial [8], antiinflammatory [9], anticonvulsant [10], muscle relaxant [11], anti-HIV and anticancer activity [12-16], and significant activity in hypertension and Alzheimer's disease [17, 18]. Consequently, several protocols for the synthesis of isoxazoles and spirooxindoles have been reported [19-28].

$( \pm)$-Camphor-10-sulfonic acid (CSA) is an effective, safe, water-soluble, and reusable organocatalyst for various organic reactions, for instance, Friedel-Crafts reactions [29], synthesis of dioxabicyclo[3.3.1]nonane and $\beta$-amino naphthol compounds [30,31], and synthesis of spirocyclic compounds [32], and is also frequently used in the optical resolution of amines [33].
It is important to develop new synthetic processes that provide better environmental performance in synthetic organic chemistry [34]. Numerous organic reactions can be effectively achieved in higher yields, shorter reaction times, and milder reaction conditions under ultrasonic irradiation due to cavitational collapse [35-40].

This work aims to study the synthesis of isoxazolopyridines and spirooxindoles under ultrasonic irradiation in the presence of $( \pm)$-CSA as an organocatalyst. Antioxidants are being increasingly noticed for the prevention of the damaging influence of free radicals in the human body [41]. Hence, to expand biological curiosity to isoxazolopyridine and spirooxindole compounds, the new synthesized compounds were tested for their free radical scavenging activity (determined for DPPH), reducing activity (reduction of the $\mathrm{Fe}^{3+}$ /ferricyanide complex to its ferrous form), metal chelating (chelating activity capacity of ferrous ions) activity, superoxide scavenging activity, and total antioxidant activity.

\section{Experimental}

2.1. Materials and Methods. NMR spectra were determined on a Bruker Avance III-500 MHz NMR. Chemical shifts are given in ppm downfield from $\mathrm{Me}_{4} \mathrm{Si}$ in DMSO- $d_{6}$ solution. Coupling constants are given in Hz. The FTIR spectra were 
<smiles>Cc1cc(N)on1</smiles>

SCHEME 1: Three-component synthesis of isoxazolopyridine derivatives.<smiles>O=C1Nc2ccccc2C1=O</smiles>

6<smiles>CCCCCCCCCCC</smiles>

2<smiles>CC(Cl)(Cl)CI1CCC(=O)CC1CCO</smiles>

7<smiles></smiles>

8

Scheme 2: Three-component synthesis of spirooxindole derivatives.

recorded on a Perkin-Elmer FT-IR spectrometer (ATR) and absorption frequencies are reported in $\mathrm{cm}^{-1}$. MS spectra were recorded on an AB Sciex 3200 QTRAP LC-MS/MS. Elemental analyses were measured with CHNS-932 LECO apparatus and were in good agreement $( \pm 0.2 \%)$ with the calculated values. Ultrasonication was performed in an Alex Ultrasonic Bath with a frequency of $32 \mathrm{kHz}$ and a power of $230 \mathrm{~W}$. The internal dimensions of the ultrasonic cleaner tank were $240 \times 140 \times 100 \mathrm{~mm}$ with liquid holding capacity of $3 \mathrm{~L}$. The reactor was a $100 \mathrm{~mL}$ Pyrex round-bottom flask. The reaction flasks were suspended in the center of the bath, and the addition or removal of water controlled the temperature of the water bath. Melting points were measured on a Gallenkamp melting-point apparatus. TLC was conducted on standard conversion aluminum sheets precoated with a $0.2 \mathrm{~mm}$ layer of silica gel. All reagents were commercially available.

2.2. General Procedure for the Synthesis of Isoxazolopyridine Derivatives $(\mathbf{4 a}-\boldsymbol{e}$ and $\mathbf{5} \boldsymbol{f}-\boldsymbol{h})$ under Ultrasonic Irradiation. A mixture of CSA (34.8 mg, $0.15 \mathrm{mmol}), 5$-amino-3methylisoxazole, $(1.00 \mathrm{mmol})$, indan-1,3-dione $(1.00 \mathrm{mmol})$, and aromatic aldehydes $(1.00 \mathrm{mmol})$ in $5 \mathrm{~mL}$ EtOH was irradiated with ultrasound of low power at $70^{\circ} \mathrm{C}$ for the period of time indicated in Scheme 1 and Table 2. After completion of the reaction, as indicated by TLC monitoring, the resultant solid was washed with water and crystallized from ethanol to give products $\mathbf{4 a - e}$ and $\mathbf{5 f}-\mathbf{h}$.

2.3. General Procedure for the Synthesis of Spirooxindole Derivatives $(\mathbf{8 a}-\boldsymbol{d})$ under Ultrasonic Irradiation. A mixture of CSA $(23.2 \mathrm{mg}, 0.10 \mathrm{mmol}), 5$-amino-3-methylisoxazole, $(1.00 \mathrm{mmol})$, isatin $(1.00 \mathrm{mmol})$, and $\beta$-diketones $(1.00 \mathrm{mmol})$ in $5 \mathrm{~mL}$ EtOH was irradiated with ultrasound of low power at $70^{\circ} \mathrm{C}$ for the period of time indicated in Scheme 2 and Table 3 . After completion of the reaction, as indicated by TLC monitoring, the resultant solid was washed with water and crystallized from ethanol to give products 8a-d.
2.4. Free Radical Scavenging Activity. The free radical scavenging activity was determined by 1,1-diphenyl-2-picrylhydrazyl $\left(\mathrm{DPPH}^{\circ}\right)$. The activity was assayed according to the methodology described by Brand-Williams et al. [42]. $20 \mathrm{mg} / \mathrm{L} \mathrm{DPPH}^{\circ}$ in methanol was prepared and $1.5 \mathrm{~mL}$ of this solution was added to $0.75 \mathrm{~mL}$ of isoxazolopyridine or spirooxindole compounds solution in water at various concentrations $(25-400 \mu \mathrm{g} / \mathrm{mL})$. After 30 minutes the absorbance was measured at $517 \mathrm{~nm}$. The inhibition activity percentage was calculated according to the following equation:

$$
\text { Free radical scavenging effect } \%=\left[\frac{\left(A_{0}-A_{1}\right)}{A_{0}}\right] \cdot 100
$$

( $A_{0}$ is the control absorbance and $A_{1}$ is the sample solution absorbance).

2.5. The Reducing Power Assay. The reducing power of newly synthesized compounds was assayed according to the methodology of Oyaizu [43]. Various concentrations of compounds $(25-400 \mu \mathrm{g} / \mathrm{mL})$ in $1 \mathrm{~mL}$ of distilled water were mixed with $0.2 \mathrm{M}$ phosphate buffer and $2.5 \mathrm{~mL}$ of potassium ferricyanide $\left[\mathrm{K}_{3} \mathrm{Fe}(\mathrm{CN})_{6}\right]$. This mixture was incubated at $50^{\circ} \mathrm{C}$ for 30 minutes and trichloroacetic acid $(2.5 \mathrm{~mL}, 10 \%$, $\mathrm{w} / \mathrm{v})$ was added to the mixture and then centrifuged at $3000 \mathrm{rpm}$ for $10 \mathrm{~min}$. Lastly, $2.5 \mathrm{~mL}$ of solution was mixed with $0.5 \mathrm{~mL} \mathrm{FeCl}_{3}$ and distilled water $(2.5 \mathrm{~mL})$ and then the absorbance was measured at $700 \mathrm{~nm}$.

2.6. Metal Chelating Activity. The ferrous ions' $\left(\mathrm{Fe}^{2+}\right)$ chelating activities of the isoxazolopyridines, spirooxindoles, and standards were investigated according to the methodology described by Decker and Welch [44]. $1 \mathrm{~mL}$ of various concentrations $(25-400 \mu \mathrm{g} / \mathrm{mL})$ of the newly synthesized compounds was mixed with $3.7 \mathrm{~mL}$ of water. This mixture was incubated for 30 minutes with $2 \mathrm{mM} \mathrm{FeCl}_{2}$. Afterward, $5 \mathrm{mM}$ ferrozine was added and left standing at room temperature 
for $10 \mathrm{~min}$. The absorbance of the resulting solution was measured at $562 \mathrm{~nm}$.

$$
\text { Metal chelating activity }(\%)=\left[\frac{\left(A_{0}-A_{1}\right)}{A_{0}}\right] \cdot 100 \text {. }
$$

2.7. Superoxide Scavenging Activity. The superoxide anion scavenging activity of isoxazolopyridine and spirooxindole compounds was done according to the methodology described by Liu et al. [45]. The superoxide radicals were formed in $3 \mathrm{~mL}$ of $16 \mathrm{mM}$ Tris- $\mathrm{HCl}$ buffer which contained $1 \mathrm{~mL}$ of $50 \mu \mathrm{M}$ NBT solution, $1 \mathrm{~mL}$ of $78 \mu \mathrm{M}$ NADH solution, and a sample solution of the newly synthesized compounds $(25-400 \mu \mathrm{g} / \mathrm{mL})$ in water. $1 \mathrm{~mL}$ of $10 \mu \mathrm{M}$ phenazine methosulphate (PMS) solution was added to the mixture. Then the mixture was incubated at $25^{\circ} \mathrm{C}$ for $5 \mathrm{~min}$ and the absorbance at $560 \mathrm{~nm}$ was measured.

$$
\% \text { Inhibition }=\left[\frac{\left(A_{0}-A_{1}\right)}{A_{0}}\right] \cdot 100 .
$$

2.8. Total Antioxidant Activity Assay. The total antioxidant activity of isoxazolopyridine and spirooxindole compounds was assayed according to the thiocyanate method described by Mitsuda et al. [46]. The solution of isoxazolopyridine and spirooxindole compounds $(150 \mu \mathrm{g} / \mathrm{mL})$ in $2.5 \mathrm{~mL}$ of $0.04 \mathrm{M}$ potassium phosphate buffer was added to $2.5 \mathrm{~mL}$ of linoleic acid emulsion in $0.04 \mathrm{M}$ potassium phosphate buffer. This mixed solution $(5 \mathrm{~mL})$ was incubated at $37^{\circ} \mathrm{C}$. For the period of incubation, $0.1 \mathrm{~mL}$ of the mixture was diluted with $3.7 \mathrm{~mL}$ of methanol at regular terms, and then $0.1 \mathrm{~mL}$ of $30 \%$ ammonium thiocyanate and $0.1 \mathrm{~mL}$ of $20 \mathrm{mM}$ ferrous chloride in $3.5 \%$ hydrochloric acid were added. The absorbance was measured at $500 \mathrm{~nm}$. This step was repeated every $10 \mathrm{~h}$ until the control reached its maximum absorbance value.

Inhibition of lipid peroxidation (\%)

$$
=\left[\frac{\left(A_{0}-A_{1}\right)}{A_{0}}\right] \cdot 100 .
$$

3-Methyl-4-phenyl-4H-indeno[1,2-b]isoxazolo[4,5-e]pyridin$5(10 H)$-one $(4 a)$. Red powder (EtOH), Yield (71\%), m.p. $242-244^{\circ} \mathrm{C}$; IR (ATR), $v_{\max } / \mathrm{cm}^{-1}$ : $3432(\mathrm{NH}), 3164,3068$ (arom. CH), 2857 (aliph. CH), $1699(\mathrm{C}=\mathrm{O}) ;{ }^{1} \mathrm{H}$ NMR $\left(\mathrm{DMSO}-d_{6}\right) \delta / \mathrm{ppm}: 1.86$ (s, 3H, Me), $5.11(\mathrm{~s}, 1 \mathrm{H}, \mathrm{CH}), 7.21$ (d, $1 \mathrm{H}, J=7.00 \mathrm{~Hz}, \mathrm{Ar}-\mathrm{H}), 7.29-7.46(\mathrm{~m}, 4 \mathrm{H}, \mathrm{Ar}-\mathrm{H}), 7.50-7.75$ (m, 4H, Ar-H), 12.10 (s, $1 \mathrm{H}, \mathrm{NH}) ;{ }^{13} \mathrm{C}$ NMR (DMSO-d 6 ):

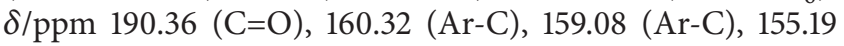
(Ar-C), 144.27 (Ar-C), 140.72 (Ar-C), 136.05 (Ar-CH), 133.39 (Ar-CH), 130.34 (Ar-CH), 129.58 (Ar-CH), 128.28 (Ar-CH), 127.84 (Ar-CH), 126.59 (Ar-CH), 123.59 (Ar-CH), 120.49 (Ar$\mathrm{CH}), 119.22$ (Ar-C), 108.28 (Ar-C), 97.40 (Ar-C), 34.48 (CH), $9.90(\mathrm{Me})$; MS: $m / z$ (ESI) $316.4[\mathrm{M}++2], 314.1[\mathrm{M}+]^{+}, 298.4$ $[\mathrm{M}+-16], 106.0[\mathrm{M}+-208]$. Anal. Calcd for $\mathrm{C}_{20} \mathrm{H}_{14} \mathrm{~N}_{2} \mathrm{O}_{2}: \mathrm{C}$, 76.42; H, 4.49; N, 8.91. Found: C, 76.50; H, 4.53; N, 8.86.

4-(3-Methyl-5-oxo-5,10-dihydro-4H-indeno[1,2-b]isoxazolo[4, 5-e]pyridin-4-yl)benzonitrile (4b). Red powder (EtOH), Yield
(76\%), m.p. $257-259^{\circ} \mathrm{C}$; IR (ATR) $v_{\max } / \mathrm{cm}^{-1}: 3432(\mathrm{NH})$, 3151, 3106 (arom. CH), 2997, 2920 (aliph. CH), $2231(\mathrm{C} \equiv \mathrm{N})$, $1713(\mathrm{C}=\mathrm{O}) ;{ }^{1} \mathrm{H}$ NMR (DMSO- $\left.d_{6}\right): \delta / \mathrm{ppm} 1.82$ (s, 3H, Me), 5.09 (s, 1H, CH), 7.25 (d, 1H, J = 7.00 Hz, Ar-H), 7.36-7.56 (m, 4H, Ar-CH), 7.77-7.82 (m, 3H, Ar-H), 12.18 (s, 1H, $\mathrm{NH}) ;{ }^{13} \mathrm{C}$ NMR (DMSO- $\left.d_{6}\right): \delta / \mathrm{ppm} 190.20(\mathrm{C}=\mathrm{O}), 160.64$ (Ar-C), 158.96 (Ar-C), $155.71(\mathrm{C} \equiv \mathrm{N}), 149.47$ (Ar-C), 135.84 (Ar-C), 133.27 (Ar-C), 132.36 (Ar-CH), 132.01 (Ar-CH), 131.81 (Ar-CH), 130.52 (Ar-CH), 130.09 (Ar-CH), 129.02 (Ar-CH), 120.60 (Ar-CH), 119.43 (Ar-CH), 118.76 (Ar-C), 109.52 (Ar-C), 107.18 (Ar-C), 96.51 (Ar-C), 34.62 (CH), 9.88 (Me); MS: $m / z$ (ESI) $338.0[\mathrm{M}+-1], 321.8$ [M+-18], 296.6 [M+-43], 221.3 [M+-118], 138.2 [M+-201], 126.3 [M+-213]. Anal. Calcd for $\mathrm{C}_{21} \mathrm{H}_{13} \mathrm{~N}_{3} \mathrm{O}_{2}$ : C, 74.33; $\mathrm{H}, 3.86 ; \mathrm{N}, 12.38$. Found: C, 74.40; H, 5.82; N, 12.43.

4-(4-(Benzyloxy)phenyl)-3-metyl-4H-indeno[1,2-b]isoxazolo[4,5-e]pyridin-5(10H)-one (4c). Red powder (EtOH), Yield (78\%), m.p. $285-287^{\circ} \mathrm{C}$; IR (ATR) $v_{\max } / \mathrm{cm}^{-1}: 3322$ (NH), 3106, 3040 (arom. CH), 2985 (aliph. CH), $1704(\mathrm{C}=\mathrm{O})$; ${ }^{1} \mathrm{H}$ NMR (DMSO-d $)$ ): $\delta / \mathrm{ppm} 2.07$ (s, 3H, Me), 4.96 (s, 1H, $\mathrm{CH}), 5.22$ (s, 2H, $\mathrm{CH}_{2}$ ), 7.17-7.44 (m, 5H, Ar-H), 7.52-7.67 (m, 4H, Ar-H), 7.75-7.97 (m, 4H, Ar-H), 12.00 (s, 1H, NH); ${ }^{13} \mathrm{C}$ NMR (DMSO- $\left.d_{6}\right): \delta / \mathrm{ppm} 189.75(\mathrm{C}=\mathrm{O}), 167.28$ (Ar-C), 159.60 (Ar-C), 157.21 (Ar-C), 147.12 (Ar-C), 145.64 (Ar-CH), 140.67 (Ar-C), 136.95 (Ar-C), 136.27 (Ar-C), 135.89 (Ar-C), 132.68 (Ar-CH), 131.08 (Ar-CH), 130.17 (Ar-CH), 128.46 (Ar$\mathrm{CH}), 127.92$ (Ar-CH), 127.65 (Ar-CH), 123.57 (Ar-CH), 122.65 (Ar-CH), 121.59 (Ar-CH), 115.24 (Ar-CH), 114.34 (Ar-CH), 113.99 (Ar-CH), 111.89 (Ar-C), 97.54 (Ar-C), $69.45\left(\mathrm{CH}_{2}\right)$, $33.61(\mathrm{CH}), 12.64(\mathrm{Me})$; MS: $m / z$ (ESI) $421.5[\mathrm{M}++1], 404.2$ [M+-16], $314.0[\mathrm{M}+-106]$. Anal. Calcd for $\mathrm{C}_{27} \mathrm{H}_{20} \mathrm{~N}_{2} \mathrm{O}_{3}$ : C, 77.13; H, 4.79; N, 6.66. Found: C, 77.21; H, 4.73; N, 6.69.

4-(2,4-Difluorophenyl)-3-methyl-4H-indeno[1,2-b]isoxazolo[4, 5-e]pyridin-5(10H)-one (4d). Red powder (EtOH), Yield (73\%), m.p. $292-294^{\circ} \mathrm{C}$; IR (ATR) $v_{\max } / \mathrm{cm}^{-1}: 3377(\mathrm{NH})$, 3101, 3052 (arom. CH), 2924 (aliph. CH), $1727(\mathrm{C}=\mathrm{O}) ;{ }^{1} \mathrm{H}$ NMR (DMSO- $\left.d_{6}\right): \delta /$ ppm $1.84(\mathrm{~s}, 3 \mathrm{H}, \mathrm{Me}), 5.19(\mathrm{~s}, 1 \mathrm{H}, \mathrm{CH})$, 6.98-7.02 (m, 2H, Ar-H), 7.15-7.27 (m, 2H, Ar-H), 7.33-7.57 (m, 3H, Ar-H), 12.15 (s, $1 \mathrm{H}, \mathrm{NH}) ;{ }^{13} \mathrm{C}$ NMR (DMSO- $d_{6}$ ): $\delta /$ ppm 190.08 (C=O), 160.62 (Ar-C), 158.75 (Ar-C), 155.81 (Ar-C), 135.94 (Ar-C), 133.33 (Ar-C), 131.99 (Ar-CH), 131.55 (Ar-CH), 130.48 (Ar-CH), 127.01 (Ar-CH), 123.81 (Ar-CH), 120.58 (Ar-CH), 119.34 (Ar-CH), 111.83 (Ar-C), 111.66 (Ar-C), 106.69 (Ar-C), 103.61 (Ar-C), 96.27 (Ar-C), 27.45 (CH), 9.53 (Me); MS: $m / z$ (ESI) $351.4[\mathrm{M}++1], 335.0[\mathrm{M}+-15], 221.1$ [M+-129]. Anal. Calcd for $\mathrm{C}_{20} \mathrm{H}_{12} \mathrm{~F}_{2} \mathrm{~N}_{2} \mathrm{O}_{2}$ : C, 68.57; $\mathrm{H}, 3.45$; N, 8.00. Found: C, 68.64; H, 3.48; N, 8.07.

3-Methyl-4-(thiophen-2-yl)-4H-indeno[1,2-b]isoxazolo[4,5e]pyridin-5(10H)-one (4e). Red powder (EtOH), Yield (78\%), m.p. 281-283 ${ }^{\circ}$; IR (ATR) $v_{\max } / \mathrm{cm}^{-1}$ : $3336(\mathrm{NH}), 3120$, 3047 (arom. CH), 2928 (aliph. CH), 1706 (C=O); ${ }^{1} \mathrm{H}$ NMR $\left(\mathrm{DMSO}-d_{6}\right): \delta / \mathrm{ppm} 1.86(\mathrm{~s}, 3 \mathrm{H}, \mathrm{Me}), 5.11(\mathrm{~s}, 1 \mathrm{H}, \mathrm{CH})$, 7.20-7.27 (m, 2H, Ar-H), 7.35-7.56 (m, 3H, Ar-H), 7.65-7.83 $(\mathrm{m}, 2 \mathrm{H}, \mathrm{Ar}-\mathrm{H}), 12.13$ (s, $1 \mathrm{H}, \mathrm{NH}) ;{ }^{13} \mathrm{C}$ NMR (DMSO- $d_{6}$ ): $\delta /$ ppm $190.40(\mathrm{C}=\mathrm{O}), 161.94$ (Ar-C), 159.11 (Ar-C), 149.09 (Ar-C), 136.66 (Ar-C), 136.14 (Ar-C), 131.97 (Ar-CH), 130.40 
(Ar-CH), 125.65 (Ar-CH), 123.80 (Ar-CH), 122.45 (Ar-CH), 121.98 (Ar-CH), 120.55 (Ar-CH), 119.20 (Ar-C), 107.42 (Ar-C), 96.92 (Ar-C), 37.00 (CH), 10.02 (Me); MS: $m / z$ (ESI) 321.2 $[\mathrm{M}++1], 305.0[\mathrm{M}+-15], 222.0[\mathrm{M}+-98]$. Anal. Calcd for $\mathrm{C}_{18} \mathrm{H}_{12} \mathrm{~N}_{2} \mathrm{O}_{2} \mathrm{~S}$ : C, 67.48; H, 3.78; N, 8.74. Found: C, 67.53; H, $3.83 ; \mathrm{N}, 8.79$.

3-Methyl-4-(3-phenoxyphenyl)-5H-indeno[1,2-b]isoxazolo[4, 5-e]pyridin-5-one (5f). Red powder (EtOH), Yield (72\%), m.p. 279-281 ${ }^{\circ}$; IR (ATR) $v_{\max } / \mathrm{cm}^{-1}: 3105,3025$ (arom. CH), 2986 (aliph. CH), 1709 (C=O); ${ }^{1} \mathrm{H}$ NMR (DMSO- $\left.d_{6}\right): \delta / \mathrm{ppm}$ 2.04 (s, 3H, Me), 7.06-7.18 (m, 3H, Ar-H), 7.20-7.61 (m, 8H, Ar-H), 7.76-7.94 (m, 2H, Ar-H); ${ }^{13} \mathrm{C}$ NMR (DMSO- $d_{6}$ ):

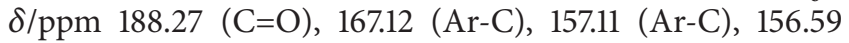
(Ar-C), 155.90 (Ar-C), 145.66 (Ar-C), 140.70 (Ar-C), 136.24 (Ar-C), 135.75 (Ar-C), 132.78 (Ar-CH), 130.08 (Ar-CH), 129.76 (Ar-CH), 124.17 (Ar-CH), 123.69 (Ar-CH), 123.55 (Ar$\mathrm{CH}), 122.01$ (Ar-CH), 121.67 (Ar-CH), 120.02 (Ar-CH), 119.53 (Ar-CH), 118.50 (Ar-CH), 111.76 (Ar-C), 12.32 (Me); MS: $m / z$ (ESI) $405.2[\mathrm{M}++1], 376.2[\mathrm{M}+-28], 361.0[\mathrm{M}+-43], 312.2$ [M+-92], $284.2[\mathrm{M}+-120]$. Anal. Calcd for $\mathrm{C}_{26} \mathrm{H}_{16} \mathrm{~N}_{2} \mathrm{O}_{3}$ : C, 77.22; H, 3.99; N, 6.93. Found: C, 77.30; H, 3.92; N, 6.97.

4-(Furan-2-yl)-3-methyl-5H-indeno[1,2-b]isoxazolo[4,5-e]pyridin-5-one (5g). Red powder (EtOH), Yield (80\%), m.p. 272-274 ${ }^{\circ}$; IR (ATR) $v_{\max } / \mathrm{cm}^{-1}: 3136,3059,3014$ (arom. $\mathrm{CH}), 2921,2874$ (aliph. CH), 1725 (C=O); ${ }^{1} \mathrm{H}$ NMR (DMSO$\left.d_{6}\right): \delta / \mathrm{ppm} 1.84(\mathrm{~s}, 3 \mathrm{H}, \mathrm{Me}), 6.93-6.95(\mathrm{~m}, 1 \mathrm{H}, \mathrm{Ar}-\mathrm{H})$, 7.57-7.97 (m, 4H, Ar-H), 8.26-8.46 (m, 2H, Ar-H); ${ }^{13} \mathrm{C}$ NMR $\left(\mathrm{DMSO}-d_{6}\right): \delta / \mathrm{ppm} 189.16(\mathrm{C}=\mathrm{O}), 150.75$ (Ar-C), 150.46 (Ar-C), 141.69 (Ar-C), 135.82 (Ar-C), 135.55 (Ar-CH), 132.79 (Ar-CH), 124.57 (Ar-CH), 124.51 (Ar-CH), 123.69 (Ar-CH), 122.86 (Ar-CH), 122.79 (Ar-CH), 121.52 (Ar-CH), 119.37 (Ar-CH), 115.00 (Ar-C), 112.98 (Ar-C), 14.04 (Me); MS: $m / z$ (ESI) $303.2[\mathrm{M}++1], 275.0$ [M+-27], 220.0 [M+-82]. Anal. Calcd for $\mathrm{C}_{18} \mathrm{H}_{10} \mathrm{~N}_{2} \mathrm{O}_{3}$ : C, 71.52; H, 3.33; N, 9.27. Found: C, 71.58; H, 3.36; N, 9.23.

3-Methyl-4-(pyridin-2-yl)-5H-indeno[1,2-b]isoxazolo[4,5e]pyridin-5-one (5h). Red powder (EtOH), Yield (73\%), m.p. $276-278^{\circ} \mathrm{C}$; IR (ATR) $v_{\max } / \mathrm{cm}^{-1}: 3106,3033$ (arom. CH), 2920 (aliph. $\mathrm{CH}), 1716(\mathrm{C}=\mathrm{O}) ;{ }^{1} \mathrm{H}$ NMR (DMSO- $\left.d_{6}\right): \delta / \mathrm{ppm}$ 2.02 (s, 3H, Me), 7.60-7.72 (m, 3H, Ar-H), 7.78-7.86 (m, 2H, Ar-H), 7.99-8.80 (m, 3H, Ar-H); ${ }^{13} \mathrm{C}$ NMR (DMSO- $\left.d_{6}\right): \delta$ 188.27 ( $\mathrm{C}=\mathrm{O}), 167.22$ (Ar-C), 157.20 (Ar-C), 149.57 (Ar-C), 149.16 (Ar-C), 144.68 (Ar-C), 140.81 (Ar-C), 136.26 (Ar-C), 136.23 (Ar-CH), 135.91 (Ar-CH), 132.89 (Ar-CH), 125.67 (Ar$\mathrm{CH}), 124.68$ (Ar-CH), 123.83 (Ar-CH), 121.89 (Ar-CH), 121.81 (Ar-CH), 111.49 (Ar-C), 12.42 (Me); MS: $m / z$ (ESI) 314.3 [M++1], $286.0[\mathrm{M}+-27], 229.0[\mathrm{M}+-84], 216.0[\mathrm{M}+-97]$. Anal. Calcd for $\mathrm{C}_{19} \mathrm{H}_{11} \mathrm{~N}_{3} \mathrm{O}_{2}: \mathrm{C}, 72.84 ; \mathrm{H}, 3.54 ; \mathrm{N}, 13.41$. Found: C, 72.91; H, 3.60; N, 13.35.

$3^{\prime}$-Methyl-7', $8^{\prime}$-dihydro-5'H-spiro[indoline-3, $4^{\prime}$-isoxazolo[5, 4-b]quinoline]-2, $5^{\prime}\left(6^{\prime} H, 9^{\prime} H\right)$-dione (8a) Lit. [22]. White powder (EtOH), Yield (80\%), m.p. 221-223 ${ }^{\circ} \mathrm{C}$; IR (ATR) $v_{\max } / \mathrm{cm}^{-1}$ : 3337, $3222(\mathrm{NH}), 3127,3028$ (arom. CH), 2955, 2934, 2887 (aliph. CH), $1732(\mathrm{C}=\mathrm{O}), 1686(\mathrm{C}=\mathrm{O}) ;{ }^{1} \mathrm{H}$ NMR (DMSO- $\left.d_{6}\right)$ : $\delta /$ ppm 1.53 (s, 3H, Me), 1.90 (dd, $\left.2 \mathrm{H}, J_{1}=13.00 \mathrm{~Hz}, J_{2}=7.00 \mathrm{~Hz}, \mathrm{CH}_{2}\right), 2.17(\mathrm{ddd}, 2 \mathrm{H}$, $\left.J_{1}=11.00 \mathrm{~Hz}, J_{2}=6.90 \mathrm{~Hz}, J_{3}=2.70 \mathrm{~Hz}, \mathrm{CH}_{2}\right), 2.64(\mathrm{t}$, $\left.2 \mathrm{H}, J=6.10 \mathrm{~Hz}, \mathrm{CH}_{2}\right), 6.80(\mathrm{~d}, 1 \mathrm{H}, J=7.70 \mathrm{~Hz}, \mathrm{Ar}-\mathrm{H})$, $6.87\left(\mathrm{dtd}, 2 \mathrm{H}, J_{1}=8.20 \mathrm{~Hz}, J_{2}=7.30 \mathrm{~Hz}, J_{3}=1.00 \mathrm{~Hz}\right.$, Ar-H), 7.13 (td, $\left.1 \mathrm{H}, J_{1}=7.50 \mathrm{~Hz}, J_{2}=1.50 \mathrm{~Hz}, \mathrm{Ar}-\mathrm{H}\right), 10.39$ $(\mathrm{s}, 1 \mathrm{H}, \mathrm{NH}), 11.08(\mathrm{~s}, 1 \mathrm{H}, \mathrm{NH}) ;{ }^{13} \mathrm{C} \mathrm{NMR}\left(\mathrm{DMSO}-d_{6}\right): \delta$ $193.70(\mathrm{C}=\mathrm{O}), 178.86(\mathrm{C}=\mathrm{O}), 159.51$ (Ar-C), 157.08 (Ar-C), 154.15 (Ar-C), 141.40 (Ar-C), 135.99 (Ar-C), 127.73 (Ar-CH), 123.28 (Ar-CH), 121.65 (Ar-CH), 109.83 (Ar-C), 108.67 (Ar-CH), 93.80 (Ar-C), $48.73(\mathrm{C}), 36.79\left(\mathrm{CH}_{2}\right), 27.39\left(\mathrm{CH}_{2}\right)$, $20.89\left(\mathrm{CH}_{2}\right), 8.78(\mathrm{Me})$; MS: $m / z$ (ESI) $322.5[\mathrm{M}++1], 279.0$ [M+-43], $251.0[\mathrm{M}+-71]$. Anal. Calcd for $\mathrm{C}_{18} \mathrm{H}_{15} \mathrm{~N}_{3} \mathrm{O}_{3}$ : C, 67.28; H, 4.71; N, 13.08. Found: C, 67.36; H, 4.65; N, 13.12 .

3-Methylspiro[indeno[2,1-e]isoxazolo[5,4-b]pyridine-4,3' -indoline $]-2^{\prime}, 5(10 H)$-dione $(\mathbf{8 b})$. Orange powder (EtOH), Yield (88\%), m.p. $242-244^{\circ} \mathrm{C}$; IR (ATR) $v_{\max } / \mathrm{cm}^{-1}: 3434,3349$ (NH), 3149, 3115, 3085 (arom. CH), 2912 (aliph. CH), 1741 $(\mathrm{C}=\mathrm{O}), 1710(\mathrm{C}=\mathrm{O}) ;{ }^{1} \mathrm{H}$ NMR $\left(\mathrm{DMSO}-d_{6}\right): \delta / \mathrm{ppm}{ }^{1} \mathrm{H}$ NMR $\left(\mathrm{DMSO}-d_{6}\right): \delta / \mathrm{ppm} 1.57(\mathrm{~s}, 3 \mathrm{H}, \mathrm{Me}), 6.70-7.10(\mathrm{~m}, 4 \mathrm{H}$, Ar-H), 7.25-7.93 (m, 4H, Ar-H), 10.63 (s, 1H, NH), 12.53 $(\mathrm{s}, 1 \mathrm{H}, \mathrm{NH}) ;{ }^{13} \mathrm{C} \mathrm{NMR}\left(\mathrm{DMSO}-d_{6}\right): \delta / \mathrm{ppm} 189.18(\mathrm{C}=\mathrm{O})$, $175.68(\mathrm{C}=\mathrm{O}), 161.52$ (Ar-C), 157.71 (Ar-C), 156.41 (Ar-C), 142.71 (Ar-C), 142.06 (Ar-C), 136.14 (Ar-CH), 132.31 (Ar-C), 130.83 (Ar-CH), 128.78 (Ar-CH), 128.13 (Ar-C), 122.67 (Ar-CH), 121.49 (Ar-CH), 120.85 (Ar-CH), 119.69 (Ar-CH), 109.85 (Ar-CH), 106.19 (Ar-C), 95.65 (Ar-C), 52.42 (C), 8.90 (Me); MS: $m / z$ (ESI) $354.1[\mathrm{M}+-1], 311.08$ [M+-43], 284.4 $\left[\mathrm{M+-71]}\right.$. Anal. Calcd for $\mathrm{C}_{21} \mathrm{H}_{13} \mathrm{~N}_{3} \mathrm{O}_{3}: \mathrm{C}, 70.98 ; \mathrm{H}, 3.69 ; \mathrm{N}$, 11.83. Found: C, 71.05; H, 3.75; N, 11.87 .

3-Methyl-5H-spiro[benzo[g]isoxazolo[5,4-b]quinoline-4,3' indoline]-2',5,10(11H)-trione (8c). Dark purple powder (EtOH), Yield (90\%), m.p. $250-252^{\circ} \mathrm{C}$; IR (ATR) $v_{\max } / \mathrm{cm}^{-1}$ : 3376, $3280(\mathrm{NH})$, 3178, 3072, 3014 (arom. CH), 2922 (aliph. $\mathrm{CH}), 1736(\mathrm{C}=\mathrm{O}), 1699(\mathrm{C}=\mathrm{O}), 1672(\mathrm{C}=\mathrm{O}) ;{ }^{1} \mathrm{H}$ NMR $\left(\mathrm{DMSO}-d_{6}\right): \delta / \mathrm{ppm} 1.62(\mathrm{~s}, 3 \mathrm{H}, \mathrm{Me}), 6.89-7.24(\mathrm{~m}, 4 \mathrm{H}$, Ar-H), 7.49-7.84 (m, 4H, Ar-H), 10.74 (s, 1H, NH), 11.83 (s, $1 \mathrm{H}, \mathrm{NH}) ;{ }^{13} \mathrm{C}$ NMR (DMSO- $\left.d_{6}\right): \delta / \mathrm{ppm} 180.99(\mathrm{C}=\mathrm{O}), 179.12$ $(\mathrm{C}=\mathrm{O}), 178.32(\mathrm{C}=\mathrm{O}), 169.42$ (Ar-C), 159.74 (Ar-C), 157.09 (Ar-C), 155.32 (Ar-C), 141.10 (Ar-C), 135.97 (Ar-C), 135.08 (Ar-CH), 133.44 (Ar-CH), 130.02 (Ar-C), 128.55 (Ar-CH), 126.11 (Ar-CH), 125.83 (Ar-CH), 124.22 (Ar-CH), 122.08 (Ar-CH), 116.06 (Ar-C), 109.17 (Ar-CH), 93.90 (Ar-C), 49.80 (C), 8.86 (Me); MS: $m / z$ (ESI) 384.1 [M++1], 341.0 [M+-43], 325.0 [M+-58], 312.0 [M+-71], 297.2 [M+-86]. Anal. Calcd for $\mathrm{C}_{22} \mathrm{H}_{13} \mathrm{~N}_{3} \mathrm{O}_{4}$ : C, 68.93; H, 3.42; N, 10.96. Found: C, 69.05; $\mathrm{H}, 3.53 ; \mathrm{N}, 11.85$.

3-Methyl-6,7-dihydrospiro[cyclopenta[e]isoxazolo[5,4-b]pyridine-4,3'-indoline]-2',5(8H)-dione $(\mathbf{8 d})$. White powder (EtOH), Yield (76\%), m.p. $216-218^{\circ} \mathrm{C}$; IR (ATR) $v_{\max } / \mathrm{cm}^{-1}$ : 3340, $3220(\mathrm{NH}), 3125,3025$ (arom. CH), 2967, 2936, 2883 (aliph. CH), $1735(\mathrm{C}=\mathrm{O}), 1689(\mathrm{C}=\mathrm{O}) ;{ }^{1} \mathrm{H}$ NMR (DMSO- $\left.d_{6}\right)$ : $\delta /$ ppm 1.52 (s, 3H, Me), $2.33\left(\mathrm{~d}, 2 \mathrm{H}, J=4.90 \mathrm{~Hz}, \mathrm{CH}_{2}\right.$ ), 2.77 (br s, $2 \mathrm{H}, \mathrm{CH}_{2}$ ), 6.83-7.00 (m, 3H, Ar-H), 7.20 (t, $1 \mathrm{H}$, $J=7.30 \mathrm{~Hz}, \mathrm{Ar}-\mathrm{H}), 10.58(\mathrm{~s}, 1 \mathrm{H}, \mathrm{NH}), 11.69$ (s, 1H, NH); ${ }^{13} \mathrm{C}$ NMR (DMSO- $\left.d_{6}\right): \delta / p p m 199.32(\mathrm{C}=\mathrm{O}), 177.45(\mathrm{C}=\mathrm{O})$, 166.86 (Ar-C), 161.74 (Ar-C), 157.46 (Ar-C), 141.44 (Ar-C), 
TABLE 1: Optimization of ( \pm -CSA catalyst loading in the synthesis of compound $\mathbf{4 a}$.

\begin{tabular}{lcccc}
\hline $\begin{array}{l}( \pm)-C S A \\
(\text { mol } \%)\end{array}$ & $\begin{array}{c}\text { Temperature } \\
\left({ }^{\circ} \mathrm{C}\right)\end{array}$ & Conditions & Time $(\min )$ & Yield $(\%)^{\mathrm{a}}$ \\
\hline- & 40 & US & 300 & 35 \\
5 & 40 & US & 180 & 52 \\
5 & 70 & US & 120 & 58 \\
10 & 70 & US & 120 & 65 \\
15 & 70 & US & 120 & 71 \\
20 & 70 & US & 120 & 72 \\
20 & 78 & $\Delta /$ reflux & 360 & 51 \\
\hline
\end{tabular}

${ }^{\mathrm{a}}$ Isolated yield.

133.79 (Ar-C), 128.44 (Ar-CH), 124.24 (Ar-CH), 122.03 (Ar-CH), 114.12 (Ar-C), 109.16 (Ar-CH), 94.37 (Ar-C), 46.85 (C), $33.55\left(\mathrm{CH}_{2}\right), 24.12\left(\mathrm{CH}_{2}\right), 8.84(\mathrm{Me}) ; \mathrm{MS}: \mathrm{m} / z$ (ESI) $308.3[\mathrm{M}++1], 265.0[\mathrm{M}+-43], 238.0$ [M+-70]. Anal. Calcd for $\mathrm{C}_{17} \mathrm{H}_{13} \mathrm{~N}_{3} \mathrm{O}_{3}$ : C, 66.44; $\mathrm{H}, 4.26 ; \mathrm{N}, 13.67$. Found: C, 66.52; $\mathrm{H}, 4.32 ; \mathrm{N}, 13.70$.

\section{Results and Discussion}

Initially, the three-component reaction of benzaldehyde (1.00 mmol), indan-1,3-dione $(1.00 \mathrm{mmol})$, and 5-amino-3methylisoxazole $(1.00 \mathrm{mmol})$ was examined under ultrasonic irradiation without a catalyst at $40^{\circ} \mathrm{C}$ in $5 \mathrm{~mL}$ EtOH. The formation of compound $\mathbf{4 a}$ was completed in 300 minutes with a yield of $35 \%$. When the same reaction was examined in the presence of $5 \mathrm{~mol} \%( \pm)$-CSA at $40^{\circ} \mathrm{C}$ under ultrasound irradiation, the product was obtained with a yield of $52 \%$ in 180 minutes (Table 1). Increasing the temperature to $70^{\circ} \mathrm{C}$ increased the yield to $58 \%$ and the reaction was completed in 120 minutes. In order to observe the effect of the amount of $( \pm)$-CSA on the reaction, experiments using different amounts of catalyst were performed (Table 1). The best result was obtained by carrying out the reaction using $15 \mathrm{~mol} \%$ of $( \pm)$-CSA at $70^{\circ} \mathrm{C}$ under ultrasonic irradiation.

Using the best optimized conditions, several isoxazolopyridine derivatives were synthesized at $70^{\circ} \mathrm{C}$ in the presence of $15 \mathrm{~mol} \%( \pm)-\mathrm{CSA}$ catalyst in EtOH under ultrasonic irradiation (Scheme 1). The results are summarized in Table 2.

It is important to point out the fact that for some unknown reasons the reaction involving 5-amino-3methylisoxazole, indan-1,3-dione, and aromatic aldehydes gave isoxazolopyridines $\mathbf{5}$ or their dihydro derivatives 4 independently of the substituent carried by the aldehydes.

Then the spirooxindole derivatives were synthesized via multicomponent reaction of 5-amino-3-methylisoxazole, isatin, and $\beta$-diketones in the presence of $( \pm)$-CSA catalyst under ultrasonic irradiation (Scheme 2). The best yields were observed in the presence of $10 \mathrm{~mol} \%( \pm)$-CSA in EtOH under ultrasonic irradiation at $70^{\circ} \mathrm{C}$ (Table 3).

The structure of the novel generated compounds was confirmed by Fourier transform-infrared (FTIR), ${ }^{1} \mathrm{H},{ }^{13} \mathrm{C}$, APT NMR techniques, and mass spectroscopy. In the ${ }^{1} \mathrm{H}$
TABLE 2: Synthesis of isoxazolopyridine derivatives in the presence of catalytic amount of $( \pm)$-CSA.

\begin{tabular}{lccc}
\hline Product & Ar & Time $(\mathrm{min})$ & ${\text { Yield }(\%)^{\mathrm{a}}}^{\mathrm{a}}$ \\
\hline $\mathbf{4 a}$ & Phenyl & 120 & 71 \\
$\mathbf{4 b}$ & 4-Cyanophenyl & 120 & 76 \\
$\mathbf{4 c}$ & 4-Benzyloxyphenyl & 120 & 78 \\
$\mathbf{4 d}$ & 2,4-Difluorophenyl & 120 & 73 \\
$\mathbf{4 e}$ & Thiophen-2-yl & 120 & 78 \\
$\mathbf{5 f}$ & 3-Phenoxyphenyl & 120 & 72 \\
$\mathbf{5 g}$ & Furan-2-yl & 120 & 80 \\
$\mathbf{5 h}$ & Pyridine-2-yl & 120 & 73 \\
\hline
\end{tabular}

${ }^{\mathrm{a}}$ Isolated yield.

NMR spectra of the isoxazolopyridines $4 \mathbf{a}-\mathbf{e}$, benzilic C$\mathrm{H}$ proton resonated near $\delta 5.06-5.30$ and in the ${ }^{13} \mathrm{C} N M R$ spectra, the benzilic $\mathrm{C}-\mathrm{H}$ carbon resonated near $\delta 27-37$. The mass spectra of all novel compounds exhibited the expected molecular ion peak.

Generally, efficient HAT agents are the compounds which show high hydrogen atom donating ability, and they usually have low heteroatom-H bond dissociation energies. Abstraction of hydrogen from these compounds leads to Ccentered radicals which are stabilized by resonance or the generation of sterically hindered radicals [47]. In this study, the antioxidant activity of the synthesized compounds $4 \mathbf{a}-\mathbf{e}$, $\mathbf{5 f}-\mathbf{g}$, and $\mathbf{8 a - d}$ was examined for free radical scavenging activity, reducing activity, metal chelating activity, superoxide scavenging activity, and total antioxidant activity.

The $\mathrm{DPPH}^{\bullet}$ scavenging effect of isoxazolopyridine and spirooxindole compounds (4a-e, $5 \mathbf{f}-\mathbf{h}$, and $\mathbf{8 a - d})$ and standards (BHA, BHT, Trolox, and Resorcinol) was studied and compounds $\mathbf{4 a}$ and $\mathbf{8} \mathbf{a}$ were found to be the ones with the most potential activity (Figure 1). These results indicate that the new synthesized compound had a moderate effect on the scavenging free radical. A higher DPPH radical scavenging activity is associated with a lower $\mathrm{EC}_{50}$ value.

As indicated in Figure 2, the reducing power of compounds $\mathbf{5 f}, \mathbf{4 c}, \mathbf{8 a}$, and $\mathbf{4 b}$ at $400 \mu \mathrm{g} / \mathrm{mL}$ concentration is higher than the other synthesized compounds and the reducing power was increased by increasing the concentration.

The metal chelating activity for the ferrous ion of the new synthesized compounds was assayed by the inhibition of the red-colored ferrozine/ $\mathrm{FeCl}_{2}$ complex. EDTA, Trolox, and BHT were used as standard compounds. The isoxazolopyridine and spirooxindole compounds showed moderate to good metal chelating activities at 25, 50, 100, 200, and $400 \mu \mathrm{g} / \mathrm{mL}$ concentrations (Figure 3). Compound $\mathbf{4 b}$ exhibited the highest chelating activity among the tested compounds at $400 \mu \mathrm{g} / \mathrm{mL}$.

The superoxide scavenging activity of isoxazolopyridine and spirooxindole compounds was compared with the same concentrations of BHT, Trolox, and BHA. None of the compounds showed greater antioxidant activity than the standards. However, compounds $\mathbf{5 g}, \mathbf{5 h}, \mathbf{4 e}$, and $\mathbf{4 b}$ exhibited higher inhibition than the other synthesized compounds (Figure 4). 
TABLE 3: Synthesis of spirooxindole derivatives in the presence of catalytic amount of ( \pm )-CSA.

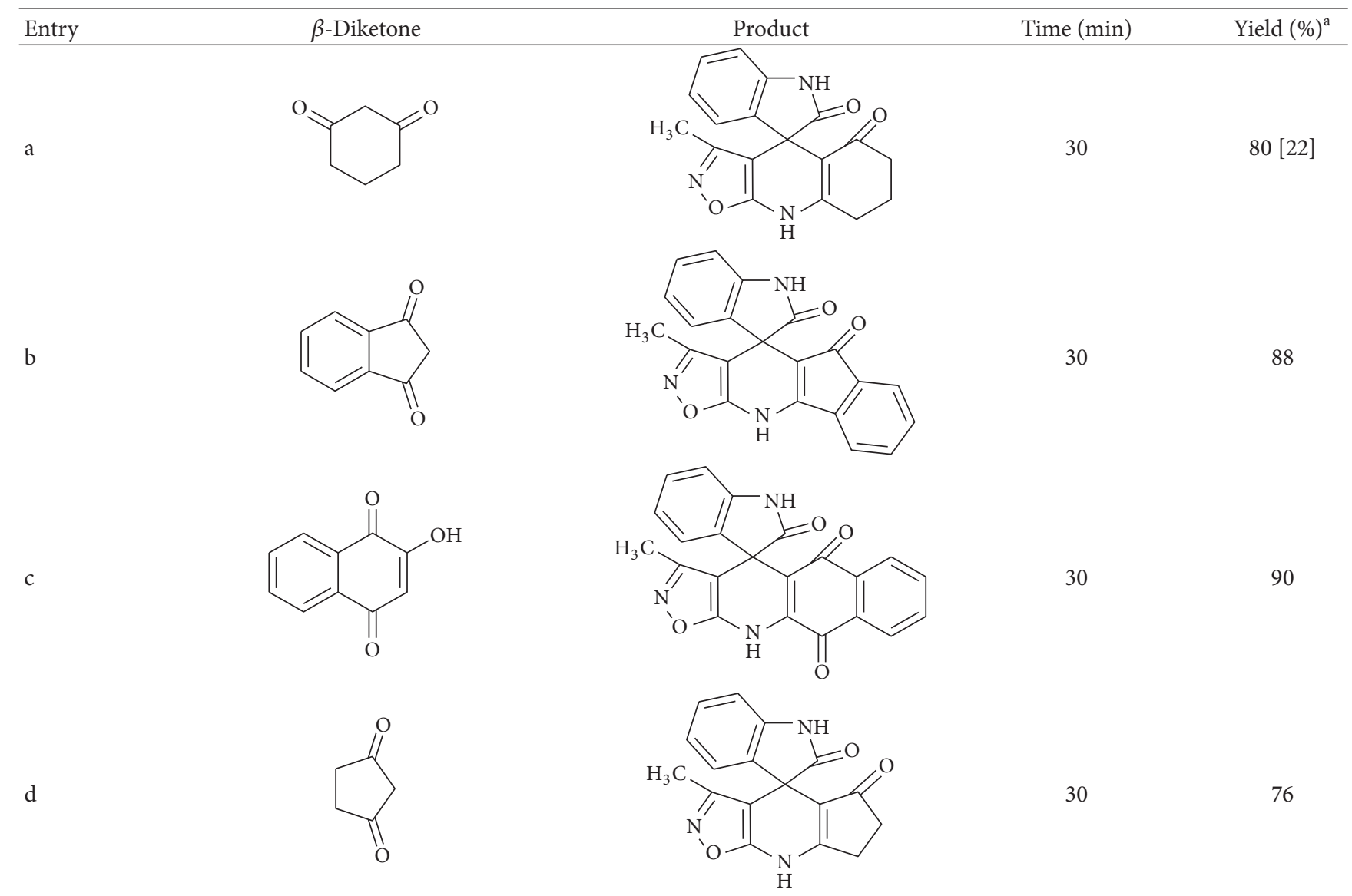

${ }^{\mathrm{a}}$ Isolated yield.

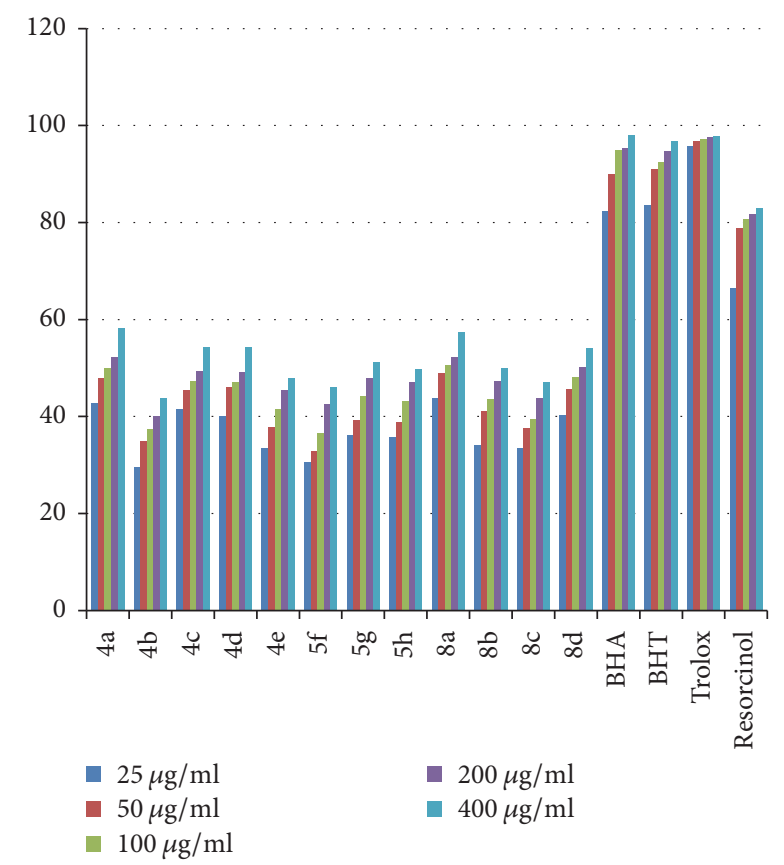

FIGURE 1: DPPH radical scavenging activity of isoxazolopyridine and spirooxindole compounds. BHA, BHT, Trolox, and Resorcinol were used as reference.

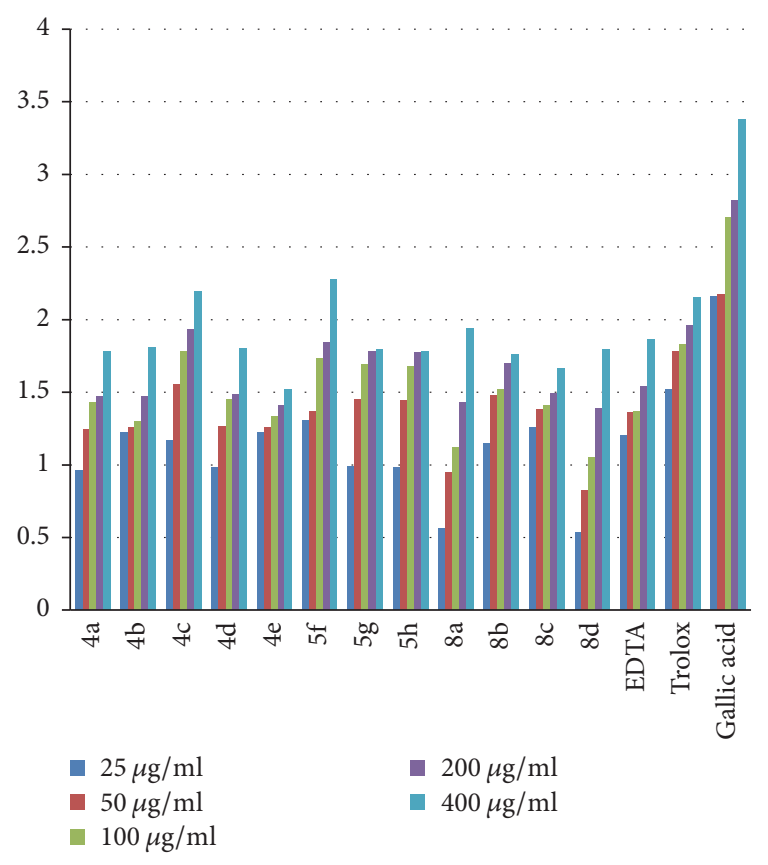

FIGURE 2: Reducing power of isoxazolopyridine and spirooxindole compounds. EDTA, Trolox, and Gallic acid were used as reference. 


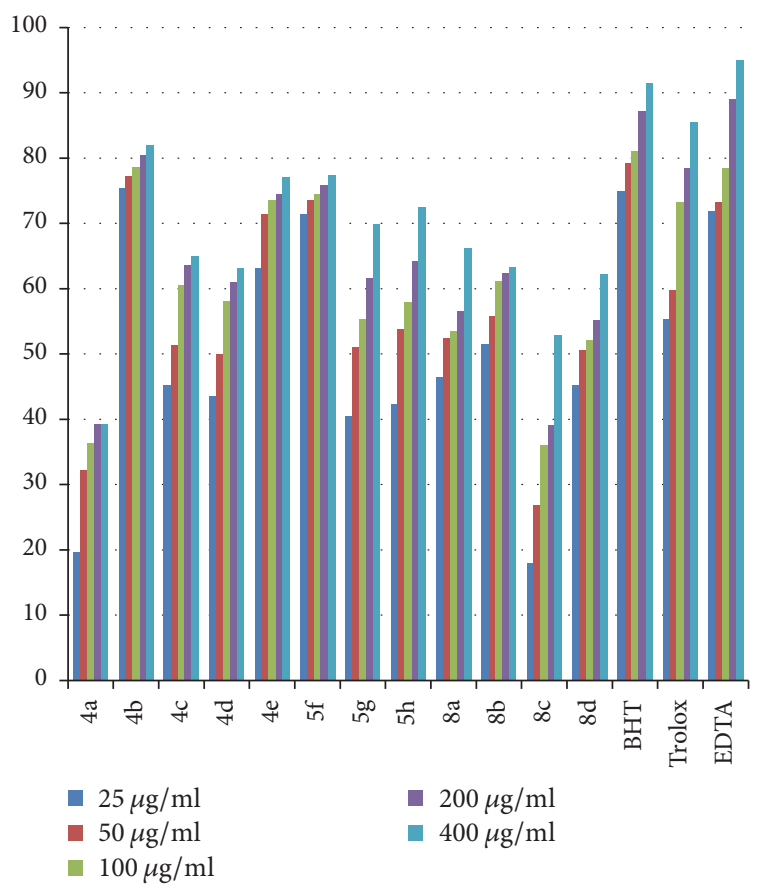

Figure 3: Metal chelating activity of isoxazolopyridine and spirooxindole compounds. BHT, Trolox, and EDTA were used as reference.

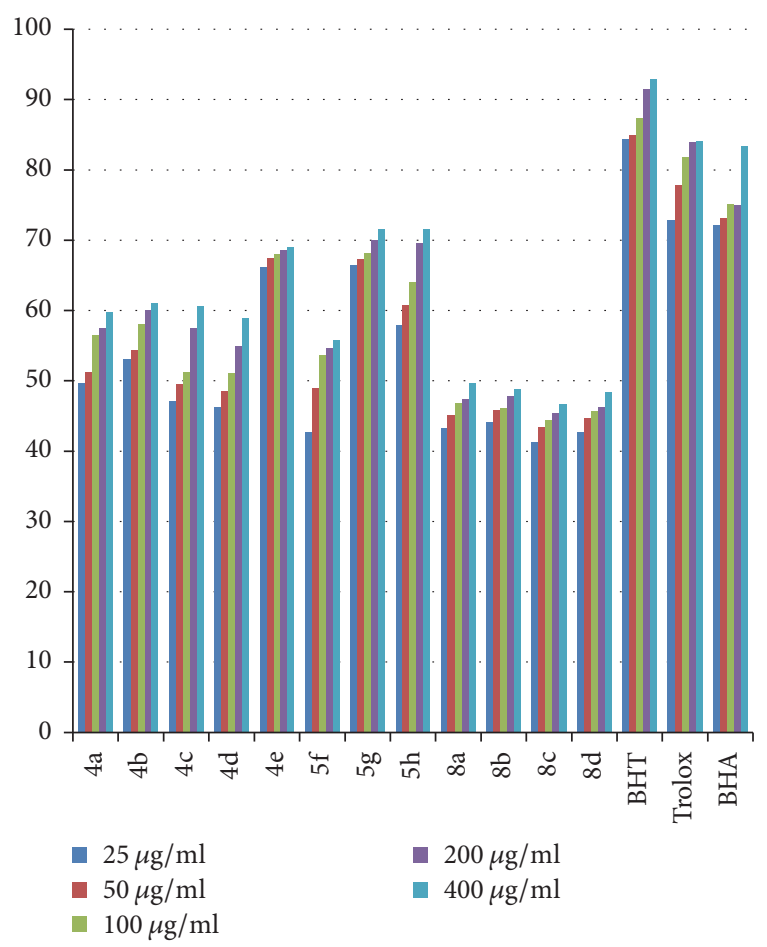

FIGURE 4: Superoxide scavenging activity of isoxazolopyridine and spirooxindole compounds. BHT, Trolox, and BHA were used as reference.

Total antioxidant activity of the isoxazolopyridine and spirooxindole compounds was assayed by thiocyanate methodology. Compounds $\mathbf{4 b}$ and $\mathbf{8 a}$ showed effectual

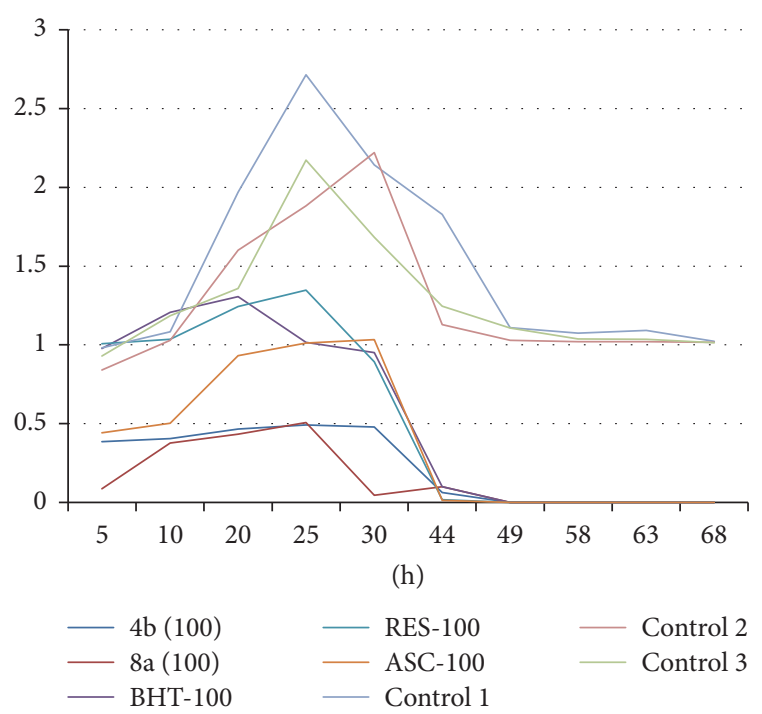

FIGURE 5: Total antioxidant activities of isoxazolopyridine and spirooxindole compounds. BHT, Resorcinol, and Ascorbic acid were used as reference.

antioxidant activity (Figure 5). The results indicated that compound $\mathbf{4 b}$ had stronger total antioxidant activity than BHT, Resorcinol, and Ascorbic acid at the same concentration $(100 \mu \mathrm{g} / \mathrm{mL})$.

\section{Conclusions}

In conclusion, new isoxazolopyridine and spirooxindole compounds were synthesized via one-pot three-component reaction of 5-amino-3-methylisoxazole, indan-1,3-dione, and aromatic aldehydes and reaction of 5-amino-3methylisoxazole, isatin, and $\beta$-diketones in the presence of $( \pm)$-camphor-10-sulfonic acid under ultrasound-promoted conditions. This procedure provided a practical, simple, and efficient way to obtain isoxazolopyridines and spirooxindoles in moderate to high yields. The antioxidant activities of these compounds were determined. Compounds $4 \mathbf{b}, \mathbf{4 e}, \mathbf{5 f}, \mathbf{5 g}, \mathbf{5 h}$, and $\mathbf{8 a}$ exhibited higher activities.

\section{Conflicts of Interest}

The author declares that there are no conflicts of interest regarding the publication of this paper.

\section{Acknowledgments}

This study was financially supported by Kirklareli University under Project no. KLUBAP 015. The author thanks Assistant Professor Dr. Melek Gul, Associate Professor Dr. Emine Bagdatli, and Assistant Professor Dr. Aliye Gediz Erturk for their guidance and helpful comments on antioxidant activity assays. 


\section{References}

[1] A. Dömling, "Recent developments in isocyanide based multicomponent reactions in applied chemistry," Chemical Reviews, vol. 106, no. 1, pp. 17-89, 2006.

[2] J. D. Sunderhaus and S. F. Martin, "Applications of multicomponent reactions to the synthesis of diverse heterocyclic scaffolds," Chemistry - A European Journal, vol. 15, no. 6, pp. 1300-1308, 2009.

[3] J. Zhu, Q. Wang, and M. Wang, Multicomponent Reactions in Organic Synthesis, Wiley-VCH, Weinheim, Germany, 2014.

[4] T. J. J. Muller, Science of, Synthesis: Multicomponent Reactions, vol. 1, Thieme, 2014.

[5] K. Ameta and A. Dandia, Multicomponent Reactions: Synthesis of Bioactive Heterocycles, CRC Press, 2017.

[6] B. M. Trost, "The atom economy-a search for synthetic efficiency," Science, vol. 254, no. 5037, pp. 1471-1477, 1991.

[7] R. P. Herrera and E. Marqués-López, Multicomponent Reactions. Concepts and Applications for Design and Synthesis, Wiley, 2015.

[8] S. S. Panda, P. V. R. Chowdary, and B. S. Jayashree, "Synthesis, antiinflammatory and antibacterial activity of novel indolylisoxazoles," Indian Journal of Pharmaceutical Sciences, vol. 71, no. 6, pp. 684-687, 2009.

[9] J. Liu, Y. Sun, X. Zhang et al., "Spirooxindoles, a potential novel class of anti-inflammatory agents," Inflammation and Cell Signaling, vol. 1, p. e372, 2014.

[10] S. J. Wittenberger, "An efficient synthesis of the cholinergic channel activator ABT-418," Journal of Organic Chemistry, vol. 61, no. 1, pp. 356-358, 1996.

[11] T. Tatee, K. Narita, S. Kurashige et al., "Isoxazole derivatives as centrally acting muscle relaxants. III. Synthesis and activity of conformationally restricted analogs," Chemical and Pharmaceutical Bulletin, vol. 35, no. 9, pp. 3676-3690, 1987.

[12] M. M. M. Santos, "Recent advances in the synthesis of biologically active spirooxindoles," Tetrahedron, vol. 70, no. 52, pp. 9735-9757, 2014.

[13] A. Kamal, P. Suresh, A. Mallareddy et al., "Synthesis of a new 4-aza-2,3-didehydropodophyllotoxin analogues as potent cytotoxic and antimitotic agents," Bioorganic and Medicinal Chemistry, vol. 19, no. 7, pp. 2349-2358, 2011.

[14] F. Shi, X. N. Zeng, X. D. Cao et al., "Design and diversityoriented synthesis of novel 1,4-thiazepen-3-ones fused with bioactive heterocyclic skeletons and evaluation of their antioxidant and cyctotoxic activities," Bioorganic \& Medicinal Chemistry Letters, vol. 22, pp. 743-746, 2012.

[15] S. Mathusalini, T. Arasakumar, K. Lakshmi et al., "Synthesis and biological evaluation of new spirooxindoles with embedded pharmacophores," New Journal of Chemistry, vol. 40, no. 6, pp. 5164-5169, 2016.

[16] B. Yu, D.-Q. Yu, and H.-M. Liu, "Spirooxindoles: Promising scaffolds for anticancer agents," European Journal of Medicinal Chemistry, vol. 97, no. 1, pp. 673-698, 2015.

[17] M. Ur Rahman, A. Rathore, A. A. Siddiqui, G. Parveen, and M. Shahar Yar, "Synthesis and antihypertensive screening of new derivatives of quinazolines linked with isoxazole," BioMed Research International, vol. 2014, Article ID 739056, 13 pages, 2014.

[18] M. Rowley, H. B. Broughton, I. Collins et al., "5-(4Chlorophenyl)-4-methyl-3-(1-(2-phenylethyl)piperidin-4yl)isoxazole: A potent, selective antagonist at human cloned dopamine D4 receptors," Journal of Medicinal Chemistry, vol. 39, no. 10, pp. 1943-1945, 1996.
[19] A. R. Lingham, J. A. Hawley, T. Greaves, N. Jackson, F. Antolasic, and H. M. Hügel, "Revisiting the three component synthesis of isoxazolo[5,4-b]pyridines, 4-aryl-3,7,7-trimethylisoxazolo[5,4-b]quinolin-5(6H)-ones and related heterocycles," Polyhedron, vol. 120, pp. 175-179, 2016.

[20] E. A. Muravyova, V. V. Tkachenko, S. M. Desenko et al., "Behavior of 5-amino-3-methylisoxazole in multicomponent heterocyclizations with carbonyl compounds under thermal heating and non-classical conditions," Arkivoc, vol. 2013, no. 3, pp. 338-371, 2013.

[21] S.-J. Tu, X.-H. Zhang, Z.-G. Han et al., "Synthesis of isoxazolo[5,4-b]pyridines by microwave-assisted multi-component reactions in water," Journal of Combinatorial Chemistry, vol. 11, no. 3, pp. 428-432, 2009.

[22] P. Yuvaraj, K. Manivannan, and B. S. R. Reddy, "Microwaveassisted efficient and highly chemoselective synthesis of oxazolo[5,4-B]quinoline-fused spirooxindoles via catalyst- and solvent-free three-component tandem Knoevenagel/Michael addition reaction," Tetrahedron Letters, vol. 56, no. 1, pp. 78-81, 2015.

[23] T. L. Pavlovska, R. G. Redkin, V. V. Lipson, and D. V. Atamanuk, "Molecular diversity of spirooxindoles. Synthesis and biological activity," Molecular Diversity, vol. 20, no. 1, pp. 299-344, 2016.

[24] D. Cheng, Y. Ishihara, B. Tan, and C. F. Barbas, "Organocatalytic asymmetric assembly reactions: Synthesis of spirooxindoles via organocascade strategies," ACS Catalysis, vol. 4, no. 3, pp. 743762, 2014.

[25] H. J. Roh, S. Y. Kim, B. K. Min, and J. N. Kim, "An efficient synthesis of dihydrofuranyl spirooxindoles from isatin-derived propargylic alcohols and 1,3-dicarbonyls," Tetrahedron Letters, vol. 58, no. 1, pp. 21-24, 2017.

[26] A. V. Moradi, "A multicomponent reaction as a versatile tool for the synthesis of spirooxindoles using $\mathrm{N}$-alkylisatins; efficient catalysis by $\mathrm{ZnO}$ nanoparticles," Journal of Chemical Research, vol. 41, no. 1, pp. 7-11, 2017.

[27] L. Yan and Y. Wang, "Recent advances in green synthesis of $3,3^{\prime}$-spirooxindoles via isatin-based one-pot multicomponent cascade reactions in aqueous medium," ChemistrySelect, vol. 1, no. 21, pp. 6948-6960, 2016.

[28] M. Esmaeilpour, J. Javidi, and M. Divar, "A green one-pot three-component synthesis of spirooxindoles under conventional heating conditions or microwave irradiation by using $\mathrm{Fe}_{3} \mathrm{O}_{4} @ \mathrm{SiO}_{2}$-imid-PMAn magnetic porous nanospheres as a recyclable catalyst," Journal of Magnetism and Magnetic Materials, vol. 423, pp. 232-240, 2017.

[29] A. Srivastava, S. Singh, and S. Samanta, “( \pm )-CSA catalyzed Friedel-Crafts alkylation of indoles with 3-ethoxycarbonyl-3hydoxyisoindolin-1-one: an easy access of 3-ethoxycarbonyl3 -indolylisoindolin-1-ones bearing a quaternary $\alpha$-amino acid moiety," Tetrahedron Letters, vol. 54, no. 11, pp. 1444-1448, 2013.

[30] X. Jiang, Z. Song, C. Xu, Q. Yao, and A. Zhang, “(D,L)10-camphorsulfonic-acid-catalysed synthesis of diaryl-fused 2,8-dioxabicyclo[3.3.1] nonanes from 2-hydroxychalcones and naphthol derivatives," European Journal of Organic Chemistry, vol. 2014, no. 2, pp. 418-425, 2014.

[31] E. Pelit and Z. Turgut, "(+)-CSA catalyzed multicomponent synthesis of 1-[(1,3-thiazol-2-ylamino)methyl]-2-naphthols and their ring-closure reaction under ultrasonic irradiation," Journal of Chemistry, vol. 2016, Article ID 9315614, 9 pages, 2016.

[32] A. Srivastava, S. M. Mobin, and S. Samanta, “( \pm )-CSA catalyzed one-pot synthesis of 6,7-dihydrospiro[indole-3, $1^{\prime}$-isoindoline]$2,3^{\prime}, 4(1 \mathrm{H}, 5 \mathrm{H})$-trione derivatives: easy access of spirooxindoles 
and ibophyllidine-like alkaloids," Tetrahedron Letters, vol. 55, no. 11, pp. 1863-1867, 2014.

[33] R. M. Kellogg, J. W. Nieuwenhuijzen, K. Pouwer et al., "Dutch resolution: separation of enantiomers with families of resolving agents. A status report," Synthesis, no. 10, pp. 1626-1638, 2003.

[34] T. J. Mason, "Sonochemistry and the environment-providing a 'green' link between chemistry, physics and engineering," Ultrasonics Sonochemistry, vol. 14, no. 4, pp. 476-483, 2007.

[35] M. Vinatoru, E. Bartha, F. Badea, and J. L. Luche, "Sonochemical and thermal redox reactions of triphenylmethane and triphenylmethyl carbinol in nitrobenzene," Ultrasonics Sonochemistry, vol. 5, no. 1, pp. 27-31, 1998.

[36] E. Pelit and Z. Turgut, "Three-component aza-Diels-Alder reactions using $\mathrm{Yb}(\mathrm{OTf})_{3}$ catalyst under conventional/ultrasonic techniques," Ultrasonics Sonochemistry, vol. 21, no. 4, pp. 16001607, 2014.

[37] J. L. Luche, Synthetic Organic Sonochemistry, Plenium Press, New York, NY, USA, 1998.

[38] C. Dong, K. Sanjay, and M. Ackmez, Handbook on Applications of Ultrasound Sonochemistry for Sustainability, CRC Press; Taylor \& Francis Group, Boca Raton, Fla, USA, 2011.

[39] E. Pelit, "CSA catalyzed multi-component synthesis of polycyclic pyrazolo[4,3-e]pyridines under ultrasonic irradiation and their antioxidant activity," Journal of the Turkish Chemical Society, Section A: Chemistry, vol. 4, pp. 631-648, 2017.

[40] E. Pelit, “ $( \pm)$-CSA catalyzed multicomponent synthesis of indeno naphthopyrans and tetrahydrobenzo[a]xanthen-11-ones under ultrasonic irradiation," Chemical Science International Journal, vol. 20, no. 1, Article ID CSIJ.35380, 8 pages, 2017.

[41] H. Sies, "Oxidative stress: oxidants and antioxidants," Experimental Physiology, vol. 82, no. 2, pp. 291-295, 1997.

[42] W. Brand-Williams, M. E. Cuvelier, and C. Berset, "Use of a free radical method to evaluate antioxidant activity," LWT-Food Science and Technology, vol. 28, no. 1, pp. 25-30, 1995.

[43] M. Oyaizu, "Studies on product of browning reaction prepared from glucose amine," Japanese Journal of Nutrition, vol. 44, pp. 307-315, 1986.

[44] E. A. Decker and B. Welch, "Role of ferritin as a lipid oxidation catalyst in muscle food," Journal of Agricultural and Food Chemistry, vol. 38, no. 3, pp. 674-677, 1990.

[45] F. Liu, V. E. C. Ooi, and S. T. Chang, "Free radical scavenging activities of mushroom polysaccharide extracts," Life Sciences, vol. 60, no. 10, pp. 763-771, 1997.

[46] H. Mitsuda, K. Yuasumoto, and K. Iwama, "Antioxidative action of indole compounds during the autooxidation of linoleic acid," Japanese Society of Nutrition and Food Science, vol. 19, pp. 210214, 1996.

[47] D. Huang, B. Ou, and R. L. Prior, "The chemistry behind antioxidant capacity assays," Journal of Agricultural and Food Chemistry, vol. 53, no. 6, pp. 1841-1856, 2005. 

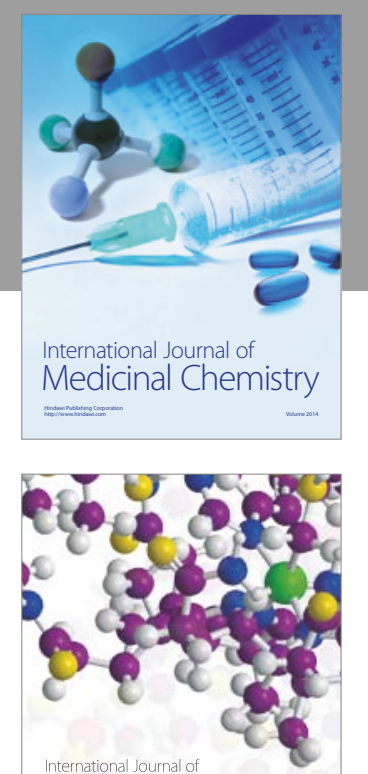

Carbohydrate Chemistry

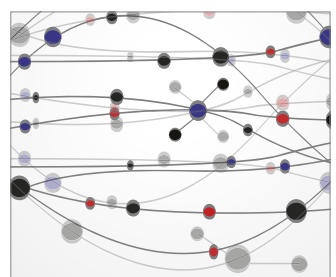

The Scientific World Journal
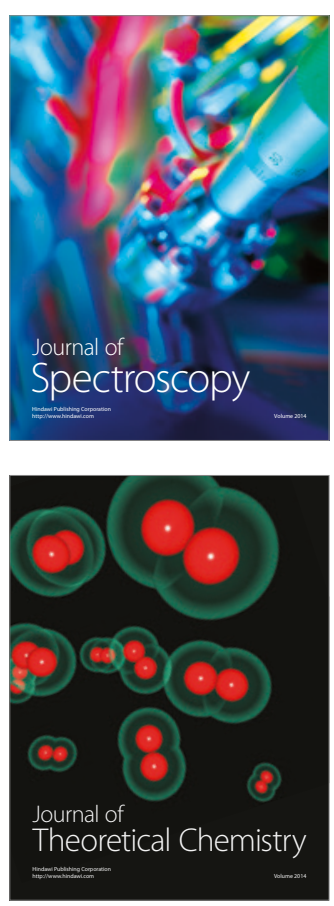
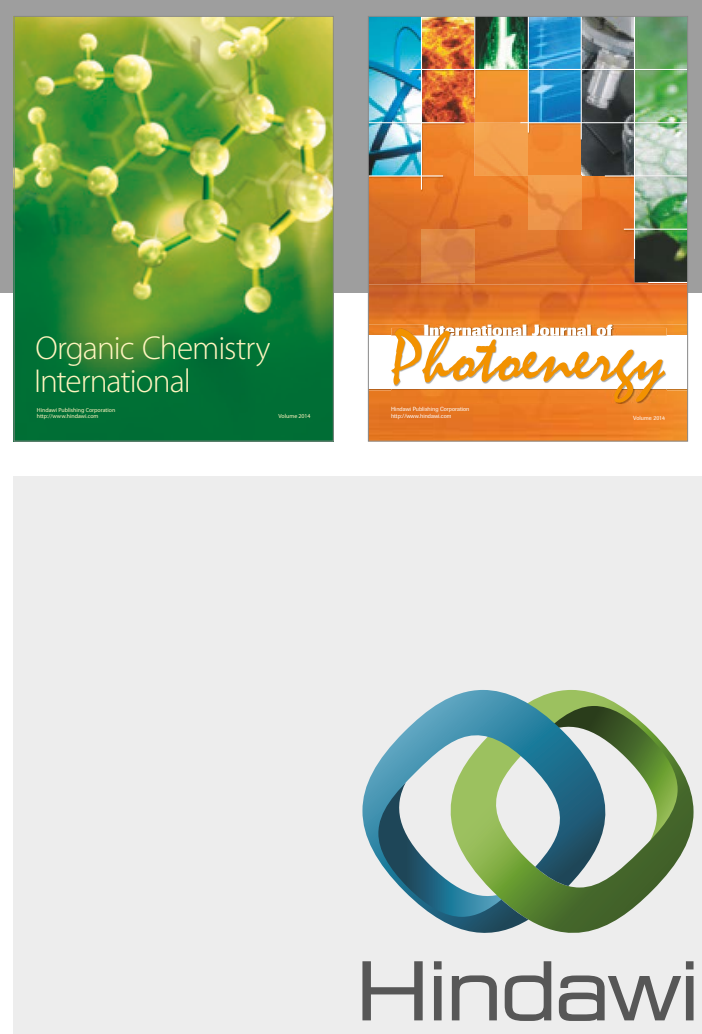

Submit your manuscripts at

https://www.hindawi.com

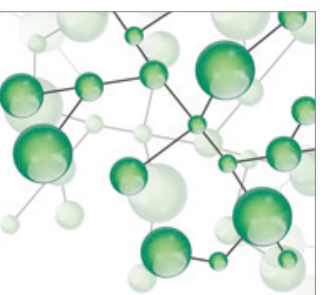

International Journal of

Inorganic Chemistry

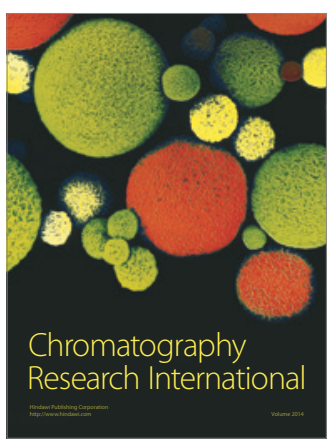

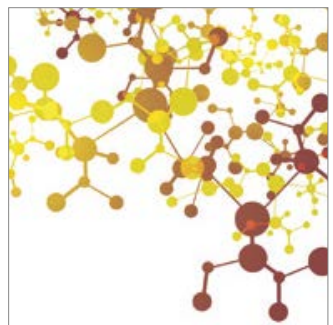

Applied Chemistry
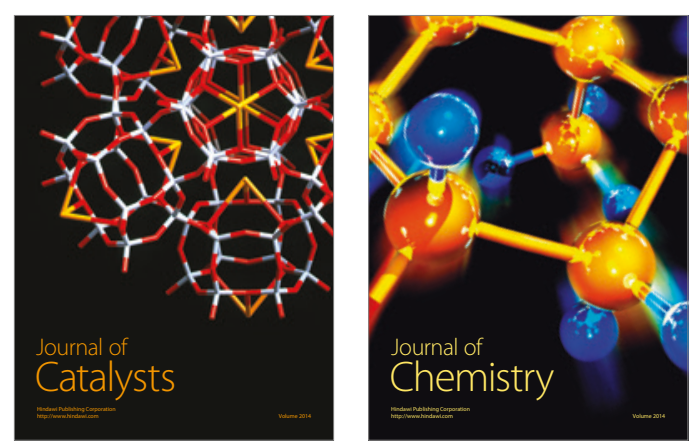
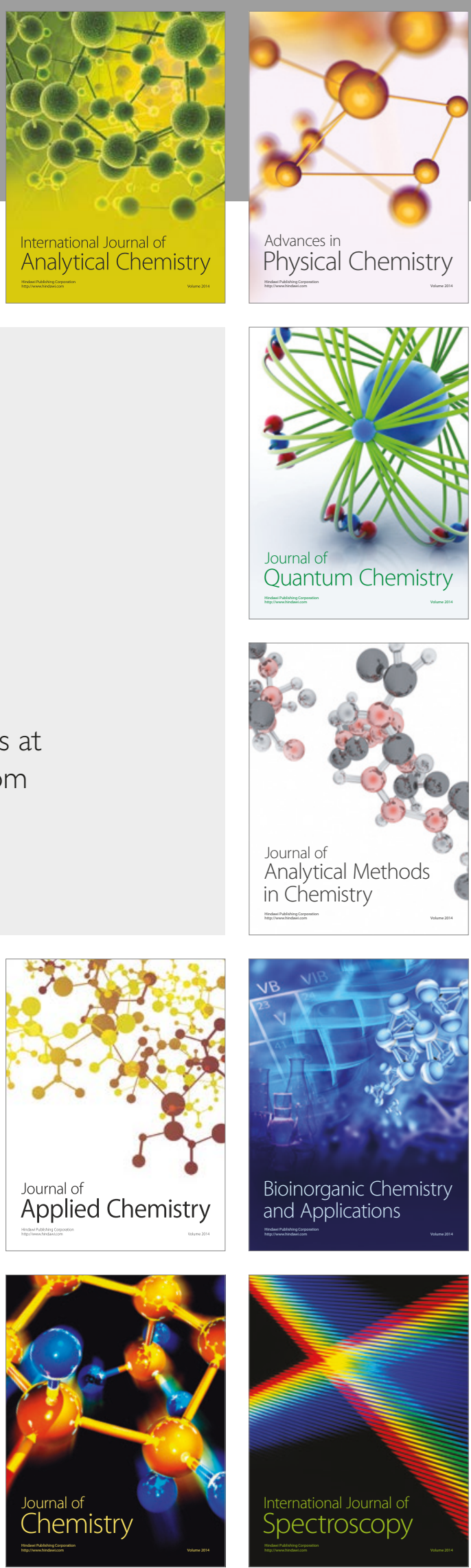